\title{
Erratum to: Parameter identification and synchronization of uncertain general complex networks via adaptive-impulsive control
}

\author{
Qunjiao Zhang · Juan Luo $\cdot$ Li Wan
}

Published online: 21 November 2013

(C) Springer Science+Business Media Dordrecht 2013

\section{Erratum to: Nonlinear Dyn (2013) 71:353-359 DOI 10.1007/s11071-012-0665-y}

In the original article [1] there are some errors in the proof of Theorem 1. Now, we point out the mistake as follows:

The inequality (9) $\left[2 \alpha \tau_{k}+\ln (\xi)<0\right.$, with $\xi>1$ and $\left.\tau_{k}>0\right]$ in Theorem 1 implies that $\alpha<0$, where $\alpha=\left(L+\frac{1}{2} \lambda-d^{*}\right)$. Thus, with the Lyapunov function (12) in [1]

$$
\begin{aligned}
V(t)= & \frac{1}{2} \sum_{i=1}^{N} e_{i}^{T}(t) e_{i}(t) \\
& +\frac{1}{2} \sum_{i=1}^{N}\left(\hat{\Theta}_{i}-\Theta_{i}\right)^{T}\left(\hat{\Theta}_{i}-\Theta_{i}\right) \\
& +\frac{1}{2} \sum_{i=1}^{N} \frac{\left(d_{i}-d_{i}^{*}\right)^{2}}{k_{i}}
\end{aligned}
$$

the enlargement $\left(L+\frac{1}{2} \lambda-d^{*}\right) \sum_{i=1}^{N} e_{i}^{T}(t) e_{i}(t) \leq$ $2 \alpha V(t)$ will not surely hold, which is appeared in the line 5 of the right column on page 356 [1].

The online version of the original article can be found under doi:10.1007/s11071-012-0665-y.

Q. Zhang $(\varangle) \cdot$ J. Luo $\cdot$ L. Wan

College of Mathematics and Computer Science, Wuhan

Textile University, Wuhan 430073, China

e-mail: qunjiao99@163.com
For correcting the mistakes in the original paper, we slightly revised them and a correct version of Theorem 1 and Corollary 1 are given in this paper.

Theorem 1 Suppose that $\boldsymbol{A 1}$ holds. Let $\lambda$ be the largest eigenvalue of $(C \otimes A)+(C \otimes A)^{T}$, if

$\beta_{i k}=\lambda_{\max }\left[\left(I+B_{i k}\right)^{T}\left(I+B_{i k}\right)\right]<1$

and there exist a constant $\xi>1$ such that

$2 \alpha \tau_{k}+\ln (\xi)<0$

under the following restriction conditions

$$
\begin{aligned}
& U_{i}=-d^{*} e_{i}(t)-\mu\|\hat{\boldsymbol{\Theta}}-\boldsymbol{\Theta}\|^{2} \frac{e_{i}}{\|E\|^{2}}, \\
& \dot{\hat{\Theta}}_{i}=-g_{i}^{T}\left(t, y_{i}(t)\right) e_{i}(t),
\end{aligned}
$$

where $d^{*}>0, \mu>0, \Theta=\left(\Theta_{1}^{T}, \Theta_{2}^{T}, \ldots, \Theta_{N}^{T}\right)^{T}$, and $\hat{\boldsymbol{\Theta}}$ is an estimation vector to $\boldsymbol{\Theta}, E(t)=\left(e_{1}^{T}(t), e_{2}^{T}(t)\right.$, $\left.\ldots, e_{N}^{T}(t)\right)^{T}$. Then, the impulsively controlled network (4) and (3) in Ref. [1] is asymptotically synchronous. Moreover,

$\hat{\Theta}_{i} \rightarrow \Theta_{i}, \quad i=1,2, \ldots, N$

Proof Construct the Lyapunov function as follows: 


$$
\begin{aligned}
V(t)= & \frac{1}{2}\|E(t)\|^{2}+\frac{1}{2}\|\hat{\boldsymbol{\Theta}}-\boldsymbol{\Theta}\|^{2} \\
= & \frac{1}{2} \sum_{i=1}^{N} e_{i}^{T}(t) e_{i}(t) \\
& +\frac{1}{2} \sum_{i=1}^{N}\left(\hat{\Theta}_{i}-\Theta_{i}\right)^{T}\left(\hat{\Theta}_{i}-\Theta_{i}\right) .
\end{aligned}
$$

Then

$$
\dot{V}(t)=\sum_{i=1}^{N} e_{i}^{T}(t) \dot{e}_{i}(t)+\sum_{i=1}^{N} \dot{\hat{\Theta}}_{i}^{T}\left(\hat{\Theta}_{i}-\Theta_{i}\right),
$$

along with error systems (6) and Assumption 1 in [1], we have

$$
\begin{aligned}
\dot{V}(t) \leq & \sum_{i=1}^{N} e_{i}^{T}(t)\left[g_{i}\left(t, y_{i}(t)\right) \cdot\left(\hat{\Theta}_{i}-\Theta_{i}\right)\right] \\
& +\sum_{i=1}^{N} L_{i} e_{i}^{T}(t) e_{i}(t) \\
& +\sum_{i=1}^{N} \sum_{j=1}^{N} e_{i}^{T}(t) c_{i j} A e_{j}(t)+\sum_{i=1}^{N} e_{i}^{T}(t) U_{i} \\
& +\sum_{i=1}^{N} \dot{\hat{\Theta}}_{i}^{T}\left(\hat{\Theta}_{i}-\Theta_{i}\right) .
\end{aligned}
$$

Denote $L=\max _{i}\left\{L_{i}\right\}$, and notice that $E(t)=$ $\left(e_{1}^{T}(t), e_{2}^{T}(t), \ldots, e_{N}^{T}(t)\right)^{T}$, substitute Eq. (10) into the above inequality, we further have

$$
\begin{aligned}
\dot{V}(t) \leq & L \sum_{i=1}^{N} e_{i}^{T}(t) e_{i}(t)+E^{T}(t)(C \otimes A) E(t) \\
& -\sum_{i=1}^{N} d^{*} e_{i}^{T}(t) e_{i}(t) \\
& -\mu \sum_{i=1}^{N}\|\hat{\boldsymbol{\Theta}}-\boldsymbol{\Theta}\|^{2} e_{i}^{T} \frac{e_{i}}{\|E\|^{2}} .
\end{aligned}
$$

In fact, $\sum_{i=1}^{N} e_{i}^{T} \frac{e_{i}}{\|E\|^{2}}=1$, thus,

$$
\dot{V}(t) \leq L \sum_{i=1}^{N} e_{i}^{T}(t) e_{i}(t)+E^{T}(t)
$$

$$
\begin{aligned}
& \times \frac{(C \otimes A)+(C \otimes A)^{T}}{2} E(t) \\
& -d^{*} \sum_{i=1}^{N} e_{i}^{T}(t) e_{i}(t)-\mu\|\hat{\boldsymbol{\Theta}}-\boldsymbol{\Theta}\|^{2} .
\end{aligned}
$$

Further,

$$
\begin{aligned}
\dot{V}(t) & \leq\left(L+\frac{1}{2} \lambda-d^{*}\right) \sum_{i=1}^{N} e_{i}^{T}(t) e_{i}(t)-\mu\|\hat{\boldsymbol{\Theta}}-\boldsymbol{\Theta}\|^{2} \\
& =\left(L+\frac{1}{2} \lambda-d^{*}\right)\|E(t)\|^{2}-\mu\|\hat{\boldsymbol{\Theta}}-\boldsymbol{\Theta}\|^{2} \\
& \leq 2 \alpha V(t),
\end{aligned}
$$

where $\alpha=\max \{v,-\mu\}<0$ and $v=L+\frac{1}{2} \lambda-d^{*}<0$ for large enough $d^{*}$.

This implies that

$V(t) \leq V\left(t_{k-1}^{+}\right) e^{2 \alpha\left(t-t_{k-1}\right)}, \quad t \in\left(t_{k-1}, t_{k}\right]$

then we get the same inequality as Eq. (13) in [1].

For $t=t_{k}$, from Eq. (7) [1], we have

$$
\begin{aligned}
V\left(t_{k}^{+}\right)= & \frac{1}{2} \sum_{i=1}^{N} e_{i}^{T}\left(t_{k}\right)\left(I+B_{i k}\right)^{T}\left(I+B_{i k}\right) e_{i}\left(t_{k}\right) \\
& +\frac{1}{2} \sum_{i=1}^{N}\left(\hat{\Theta}_{i}-\Theta_{i}\right)^{T}\left(\hat{\Theta}_{i}-\Theta_{i}\right) \\
\leq & V\left(t_{k}\right)
\end{aligned}
$$

due to $\beta_{i k}=\lambda_{\max }\left[\left(I+B_{i k}\right)^{T}\left(I+B_{i k}\right)\right]<1$.

From Eqs. (13) and (14), for $t \in\left(t_{k}, t_{k+1}\right]$, there is

$V(t) \leq V\left(t_{0}^{+}\right) e^{2 \alpha\left(t-t_{0}\right)}$.

In virtue of the inequality (9) given in Theorem 1, we know that

$e^{2 \alpha \tau_{k}}<\frac{1}{\xi}, \quad k=1,2, \ldots$

Thus, the inequality (15) can be further rewritten as

$$
\begin{aligned}
V(t) & \leq V\left(t_{0}^{+}\right)\left(e^{2 \alpha \tau_{1}}\right) \cdots\left(e^{2 \alpha \tau_{k}}\right) e^{2 \alpha\left(t-t_{k}\right)} \\
& <V\left(t_{0}^{+}\right) \frac{1}{\xi^{k}} e^{2 \alpha \tau_{k+1}},
\end{aligned}
$$

therefore $V(t) \rightarrow 0$ as $k \rightarrow \infty$ because $\xi>1$, which implies that all the errors $e_{i}(t) \rightarrow 0$ and $\hat{\Theta}_{i} \rightarrow \Theta_{i}$ $(i=1,2, \ldots, N)$. So the synchronization between the 

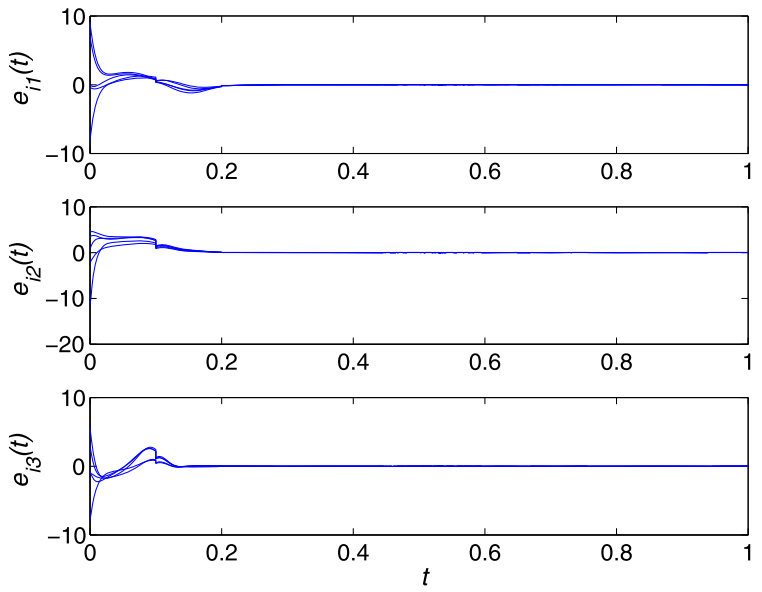

Fig. 1 Synchronization errors $e_{i}(t)(1 \leq i \leq 5)$

impulsively controlled complex network (4) and network (3) in [1] is realized and the unknown system parameters are identified simultaneously. This completes the proof.

Remark 1 After the synchronization occurs, that is, all the $e_{i j} \rightarrow 0$ as $t \rightarrow \infty$, from the error systems (6) in [1], one can get $g_{i}\left(t, y_{i}(t)\right)\left(\hat{\Theta}_{i}-\Theta_{i}\right)=0$, therefore, the conclusion (11) holds under the condition that all the column vectors of $g_{i}\left(t, y_{i}(t)\right)$ are linear independent.

Corollary 1 If a complex network consists of $N$ identical nodes, which can be described by

$\dot{x}_{i}(t)=f\left(t, x_{i}(t)\right)+g\left(t, x_{i}(t)\right) \cdot \Theta+\sum_{j=1}^{N} c_{i j} A x_{j}(t)$,

then unknown system parameters $\Theta$ can be identified by using the estimated values $\hat{\Theta}$ with the following impulsively controlled response network

$\left\{\begin{array}{l}\dot{y}_{i}(t)=f\left(t, y_{i}(t)\right)+g\left(t, y_{i}(t)\right) \cdot \hat{\Theta} \\ \quad+\sum_{j=1}^{N} c_{i j} A y_{j}(t)+U_{i}, \quad t \neq t_{k} \\ \Delta y_{i}\left(t^{+}\right)=B_{i k} e_{i}(t), \quad t=t_{k}, \quad k=1,2, \ldots \\ y_{i}\left(t_{0}^{+}\right)=y_{i 0}, \\ U_{i}=-d^{*} e_{i}(t)-\mu\|\hat{\Theta}-\Theta\|^{2} \frac{e_{i}}{\|E\|^{2}},\end{array}\right.$

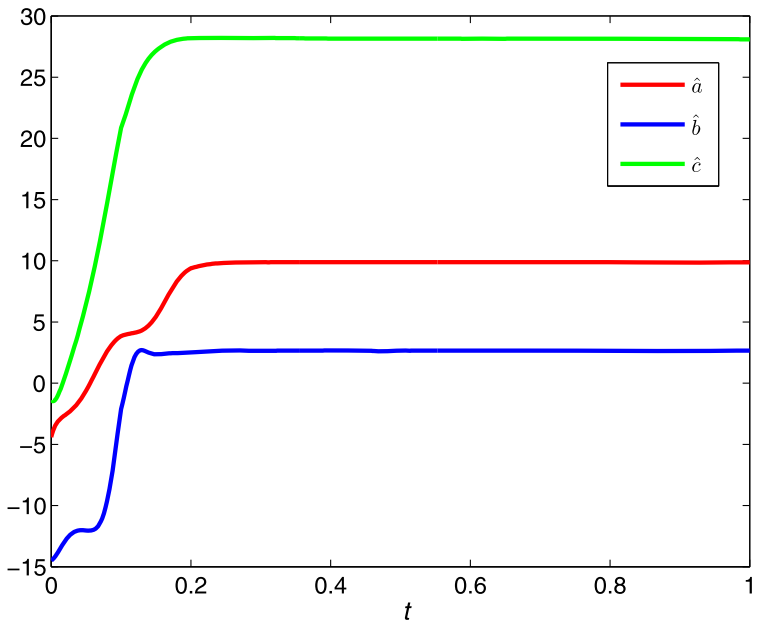

Fig. 2 Identification of system parameters

if

$2 \alpha \tau_{k}+\ln (\xi)<0$

and

$\dot{\hat{\Theta}}=-\sum_{i=1}^{N} g^{T}\left(t, y_{i}(t)\right) e_{i}(t)$,

where $\lambda$ is the largest eigenvalue of $(C \otimes A)+(C \otimes$ $A)^{T}$, the constant $\xi>1$ and $\alpha$ is defined as in Theorem 1 .

In the numerical simulation, let $d^{*}=100, \mu=1.5$, $B_{i k}=\operatorname{diag}\{-0.5,-0.5,-0.5\}, \tau_{k}=0.1$. Figures 1 and 2 , respectively show the synchronization errors of $e_{i 1}(t), e_{i 2}(t), e_{i 3}(t)$ and the identified parameters under the updating laws (20).

Acknowledgements The authors are grateful to Mr. Xiang Wei for recognizing this error and his very useful comments. This work was jointly supported by the National Natural Science Foundation of China under Grant Nos. 61304022 and 11047114.

\section{References}

1. Zhang, Q., Luo, J., Wan, L.: Parameter identification and synchronization of uncertain general complex networks via adaptive-impulsive control. Nonlinear Dyn. 71, 353-359 (2013) 